\title{
The effects of resveratrol feeding and exercise training on the skeletal muscle function and transcriptome of aged rats
}

\author{
Jing Zhou ${ }^{\text {Corresp., } 1,2}{ }^{2}$, Zhiyin Liao ${ }^{2}$, Jia Jia ${ }^{1}$, Jin-liang Chen ${ }^{2}$, Qian Xiao ${ }^{\text {Corresp. } 2}$ \\ ${ }^{1}$ Chongqing Medical and Pharmaceutical College, Chongqing, China \\ 2 Department of Geriatrics, The First Affiliated Hospital of Chongqing Medical University, Chongqing, China \\ Corresponding Authors: Jing Zhou, Qian Xiao \\ Email address: zj4321228@sina.com, xiaoqian1956@126.com
}

This study investigated the effects of resveratrol feeding and exercise training on the skeletal muscle function and transcriptome of aged rats. Male SD rats (25 months old) were divided into the control group (Old), the daily exercise training group (Trained), and the resveratrol feeding group (Resveratrol). After 6 weeks of intervention, the body mass, grip strength, and gastrocnemius muscle mass were determined, and the muscle samples were analyzed by transcriptome sequencing. The differentially expressed genes were analyzed followed by GO enrichment analysis and KEGG analysis. The Old group showed positive increases in body mass, while both the Trained and Resveratrol groups showed negative growth. No significant differences in the gastrocnemius muscle index and absolute grip strength were found among the three groups. However, the relative grip strength was higher in the Trained group than in the Old group. Only 21 differentially expressed genes were identified in the Trained group vs. the Old group, and 12 differentially expressed genes were identified in the Resveratrol group vs. the Old group. The most enriched GO terms in the Trained group vs. the Old group were mainly associated with RNA metabolic processes and transmembrane transporters, and the significantly upregulated KEGG pathways included mucin-type O-glycan biosynthesis, drug metabolism, and pyrimidine metabolism. The most enriched GO terms in the Resveratrol group vs. the Old group were primarily associated with neurotransmitter transport and synaptic vesicle, and the upregulated KEGG pathways included synaptic vesicle cycle, nicotine addiction, retinol metabolism, insulin secretion, retrograde endocannabinoid signaling, and glutamatergic synapse. Neither exercise training nor resveratrol feeding has a notable effect on skeletal muscle function and related gene expression in aged rats. However, both exercise training and resveratrol feeding have strong effects on weight loss, which is beneficial for reducing the exercise loads of the elderly. 
1 The effects of resveratrol feeding and exercise training on the skeletal muscle

2 function and transcriptome of aged rats

3

4 Jing Zhou ${ }^{1,2}$, Zhiyin $\mathrm{Liao}^{2}$, Jia Jia ${ }^{1}$, Jin-liang Chen², Qian Xiao ${ }^{2}$

5 1. Chongqing Medical and Pharmaceutical College, Chongqing, China

6 2. Department of Geriatrics, The First Affiliated Hospital of Chongqing Medical University,

7 Chongqing, China

8

9 Corresponding author ${ }^{2}$ :

10 Qian Xiao Department of Geriatrics, The First Affiliated Hospital of Chongqing Medical

11 University, Friendship Road 1, Yuan Jiagang, 400016 Chongqing, China

12 E-mail address: xiaoqian1956@126.com. 
14

\section{Abstract}

This study investigated the effects of resveratrol feeding and exercise training on the skeletal muscle function and transcriptome of aged rats. Male SD rats (25 months old) were divided into the control group (Old), the daily exercise training group (Trained), and the resveratrol feeding group (Resveratrol). After 6 weeks of intervention, the body mass, grip strength, and gastrocnemius muscle mass were determined, and the muscle samples were analyzed by transcriptome sequencing. The differentially expressed genes were analyzed followed by GO enrichment analysis and KEGG analysis. The Old group showed positive increases in body mass, while both the Trained and Resveratrol groups showed negative growth. No significant differences in the gastrocnemius muscle index and absolute grip strength were found among the three groups. However, the relative grip strength was higher in the Trained group than in the Old group. Only 21 differentially expressed genes were identified in the Trained group vs. the Old group, and 12 differentially expressed genes were identified in the Resveratrol group vs. the Old group. The most enriched GO terms in the Trained group vs. the Old group were mainly associated with RNA metabolic processes and transmembrane transporters, and the significantly upregulated KEGG pathways included mucin-type O-glycan biosynthesis, drug metabolism, and pyrimidine metabolism. The most enriched GO terms in the Resveratrol group vs. the Old group were primarily associated with neurotransmitter transport and synaptic vesicle, and the upregulated KEGG pathways included synaptic vesicle cycle, nicotine addiction, retinol metabolism, insulin secretion, retrograde endocannabinoid signaling, and glutamatergic synapse. Neither exercise training nor resveratrol feeding has a notable effect on skeletal muscle function and related gene expression in aged rats. However, both exercise training and resveratrol feeding have strong effects on weight loss, which is beneficial for reducing the exercise loads of the elderly. 
39

40

41

42

\section{Introduction}

Sarcopenia is characterized by a reduction in skeletal muscle mass and function during the aging processes (Ryall et al. 2008; Rolland et al. 2008). The pathogenesis of sarcopenia has been proposed to be caused by factors such as oxidative stress, neuromuscular dysfunction, and inflammation (Deschenes 2004; Kalinkovich and Livshits 2015; Sousa-Victor and MuñozCánoves 2016; Bano et al. 2017; Zhou et al. 2018). The mechanisms and intervention strategies of sarcopenia, however, have not been thoroughly elucidated to date (Russ et al. 2012; Kalinkovic and Livshits 2015).

Exercise training is a strategy often adopted in addressing sarcopenia because it may stimulate muscle protein synthesis, activate satellite cells, enhance muscle fiber size, reduce body fat, and improve muscle power (Rolland et al. 2008). However, most of the evidence-based data are from young or middle-aged individuals (Akune et al. 2014). Whether exercise training works well in older populations requires further study. Some previous studies also showed that exercise training can change the expression of several genes (Sakamoto et al. 2005; Thomson and Gordon 2005). Integrative studies of the effects of exercise training on both muscle function and largescale gene expression are scarce.

Oxidative stress may be involved in sarcopenia. Whether anti-oxidation can play roles in intervening sarcopenia has not been determined. Resveratrol is a naturally occurring polyphenol that has been suggested to have benefits for humans (Dolinsky and Dyck 2011). Although the effect of resveratrol on longevity or disease have been debated (Naylor 2009; Vang et al. 2011), resveratrol has been shown to protect against cellular oxidative stress, improve muscle strength and endurance (Naylor 2009; Dolinsky et al. 2012; Kaminski et al. 2012; Gordon et al. 2013), and increase exercise performance in aged animals (Murase et al. 2009). However, the molecular basis involved with the effects of resveratrol on sarcopenia has been given less attention in the literature. Studies of global gene expression in muscle are needed to evaluate the potential effects of resveratrol on sarcopenia.

Our previous works have observed that the muscle index and the relative grip strength were reduced in aged rats and suggest that the reduced expression of $5^{\prime}$ adenosine monophosphateactivated protein kinase (AMPK), insulin-like growth factor 1 (IGF-1), and calcium/calmodulindependent serine protein kinase (CASK) can explain the losses of muscle mass and function in aged rats (Liao et al. 2017; Zhou et al. 2018). The objective of the present study was to use 
RNA-Seq technology to reveal the effects of exercise training and resveratrol feeding on muscle function accompanied by global transcriptomic changes in the muscle of aged rats. The results of this study may contribute to the basal molecular information for sarcopenia interventions.

\section{Materials and Methods}

Ethics statement

All animal care and experimental procedures were approved by the Animal Care and Use Committee of Chongqing Medical University (ACUC/CMU/036/2016) and followed the standards for environmental and housing facilities for laboratory animals in China (Gb/T149252001). This study uses the method of Zhou et al. (2018), and the method description partly reproduces their wording.

Animals and treatments

Male SD rats (25 months old) were used as experimental animals. Thirty rats were randomly divided into three groups ( $\mathrm{n}=10$ for each group): one group was set as the control (Old), one group was treated with daily exercise training (Trained), and one group was treated with resveratrol feeding (Resveratrol). Animal rearing was processed as previously described in Zhou et al. (2018). Specifically, the rats were maintained in a pathogen-free room with a $12 \mathrm{~h} / 12 \mathrm{~h}$ light/dark cycle. The air temperature was controlled at $20 \pm 2{ }^{\circ} \mathrm{C}$, and the relative humidity was controlled at $55 \pm 5 \%$. All rats were fed ad libitum with standard rat chow, and diets were refreshed once every day. The Old group remained sedentary in their cages for 6 weeks. The Trained group was trained with exercise on a motorized treadmill once at 9:00 daily. The exercise training program was adapted at a speed of $15 \mathrm{~cm} \mathrm{~s}^{-1}$ for 15 min during the first week, enhanced to a speed of $20 \mathrm{~cm} \mathrm{~s}^{-1}$ for 20 min during the second week and then enhanced to $30 \mathrm{~cm}$ $\mathrm{s}^{-1}$ for 30 min during the next four weeks. During the 6 weeks, the Resveratrol group was fed with oral resveratrol at a dose of $150 \mathrm{mg} \mathrm{kg}^{-1} \mathrm{~d}^{-1}$ by mixing the resveratrol with the diet. The rats were weighed once weekly, and the ration levels were regulated.

Muscle function measurement

Muscle function was determined as previously described in Zhou et al. (2018). Specifically, at the end of the six-week period, the forelimb grip strength was determined by an electronic grip tester (Cat.47200, Ugo Basil). The strength measurement of each individual was repeated three times, and the maximal value was used as the absolute grip strength (g). The relative grip 
101 strength was calculated by dividing the absolute value $(\mathrm{g})$ by the body mass $(\mathrm{g})$. The rats were subsequently transferred back to their cages for three more days of treatment. Then, the rats were euthanized and weighed at $24 \mathrm{~h}$ after the last exercise bout/dose of resveratrol. The gastrocnemius muscles of the hindlimbs were quickly sampled and weighed to $0.01 \mathrm{~g}$. The gastrocnemius muscle index $\left(10^{-3}\right)$ was calculated by dividing the muscle mass $(\mathrm{g})$ by the body mass $(\mathrm{g})$. Then, the muscle samples from the right hindlimb were frozen in liquid nitrogen and stored in a freezer at $-80^{\circ} \mathrm{C}$ for transcriptome sequencing.

RNA-Seq library preparation and sequencing analysis

The RNA-Seq library and sequencing analysis were processed as previously described in Zhou et al. (2018). Specifically, muscle samples of three rats from each group were randomly selected for transcriptome sequencing analysis. Total RNA from the muscle tissues was isolated using TRIzol (Invitrogen) and an RNeasy RNA purification kit with DNase treatment (Qiagen). A total amount of $3 \mu \mathrm{g}$ of RNA per sample was used as an input material for the RNA sample preparations. mRNA was purified from the total RNA using poly-T oligo-attached magnetic beads and reverse transcribed into double strand cDNA fragments. Sequencing libraries were generated using the NEBNext ${ }^{\circledR}$ Ultra ${ }^{\mathrm{TM}}$ RNA Library Prep Kit from Illumina ${ }^{\circledR}$ (NEB, USA) following the manufacturer's recommendations, and index codes were added to attribute sequences to each sample. The library quality was assessed on the Agilent Bioanalyzer 2100 system. The clustering of the index-coded samples was performed on a cBot Cluster Generation System using the TruSeq PE Cluster Kit v3-cBot-HS (Illumina). The library preparations were sequenced on an Illumina HiSeq platform, and 125-bp/150-bp paired-end reads were generated.

Raw reads in the fastq format were first processed through in-house Perl scripts by removing reads containing adapters, ploy-Ns and low-quality reads from the raw data. Then, Q20, Q30, and GC content were calculated. The reference genome and gene model annotation files were downloaded from the genome website. The reads that passed the quality control were mapped to the Rattus norvegicus genome using Tophat (v2.0.12). HTSeq (v0.6.1) was used to count the read numbers mapped to each gene. Then, the FPKM of each gene was calculated based on the length of the gene, and the read count was mapped to this gene (Trapnell et al., 2010).

Differentially expressed genes and enrichment analysis

The differences in gene expression among groups were analyzed as previously described in Zhou et al. (2018). Specifically, differential expression analysis was performed using the DESeq 
132 R package (1.18.0). DESeq provided statistical routines for determining the difference in the 133 digital gene expression data using a model based on the negative binomial distribution. The resulting P-values were adjusted using Benjamini and Hochberg's approach for controlling the false discovery rate. Gene Ontology (GO) enrichment analysis of differentially expressed genes was implemented by the GOseq $\mathrm{R}$ package, in which the gene length bias was corrected. GO terms with a corrected $P$-value less than 0.05 were considered to be significantly enriched by differentially expressed genes. KOBAS software was used to test the statistical enrichment of the differentially expressed genes in the KEGG pathways.

Quantitative real-time PCR

Quantitative real-time PCR was performed as previously described in Zhou et al. (2018). Specifically, $1.5 \mu \mathrm{g}$ of extracted total RNA was added to a final volume of $20 \mu \mathrm{l}$ to synthesize cDNA using a PrimeScript ${ }^{\mathrm{TM}}$ RT Reagent Kit with gDNA Eraser (Takara Biomedical Technology, Beijing, China). Eight muscular function-related genes (Prkaa-2, FOXO3, IGF-1, CASK, TRIM67, IL-6, MGMT, SCN5a) were selected, and the coding mRNA quantity was analyzed by the ABI VIIATM7 Real-Time PCR system (Applied Biosystems, Foster City, CA) with a quantitative real-time PCR kit (GoTaq ${ }^{\circledR}$ qPCR Master Mix, Promega Biotech, Beijing, China). Amplification was performed for 1 cycle at $95{ }^{\circ} \mathrm{C}$ for $10 \mathrm{~min}$, followed by 40 cycles at $95{ }^{\circ} \mathrm{C}$ for $15 \mathrm{~s}$, and $60{ }^{\circ} \mathrm{C}$ for $30 \mathrm{~s}$ with $1 \mathrm{pM}$ primers (Table 1). qRT-PCR for all genes was performed in triplicate, with glyceraldehyde-3-phosphate dehydrogenase (GAPDH) serving as the reference gene. Relative expression levels were calculated with the $2^{-\Delta \Delta C T}$ method and were presented as fold changes compared to the Old group.

Statistical analysis

Parameters of the body and muscle were calculated using Microsoft Excel 2003 (Microsoft Corporation, Redmond, WA, USA) and analyzed using SPSS 11.5 (SPSS Inc., Chicago, IL, USA). Initial body mass, body mass gain, and specific growth rate among the groups were compared using one-way ANOVA followed by Duncan's test. Final body masses and absolute grip strengths among the groups were also compared with initial body mass as a covariate. A paired-samples t-test was used to compare the initial mass with the final body of each group. Differences were considered significant at the level of $P<0.05$. Data are presented as the mean \pm s.e.m. 


\section{Results}

164 Body mass and muscle function

165 The final body masses of the Trained group and Resveratrol group were significantly lower than

166

167

168

169

170

171

172

173

174

175

176

177

178

179

180

181

182

183 those of the Old group with initial body mass as a covariate $(P<0.001)$ (Table 2$)$. The final body masses of the Old group were $1.94 \%$ larger but not significantly different from their initial body masses $(t=0.921, P=0.388)$. Both the Trained group and the Resveratrol group showed decreases in body mass. The final body masses of the Trained group were 12.2\% lower than their initial body masses $(t=-8.763, P<0.001)$, while the final body masses of the Resveratrol group were $5.31 \%$ lower than their initial body masses $(t=-2.543, P=0.039)$. Body mass gain and specific growth rate were significantly larger in the Old group compared to both the Trained group and Resveratrol group. The gastrocnemius muscle indexes for the left limb and the right limb were $4.81 \times 10^{-3}$ and $4.88 \times 10^{-3}$ for the Old group, $5.10 \times 10^{-3}$ and $5.38 \times 10^{-3}$ for the Trained group, and $4.90 \times 10^{-3}$ and $4.97 \times 10^{-3}$ for the Resveratrol group (Fig. 1). The gastrocnemius muscle index was not significantly different among the three groups (left limb: $F=0.399, P=0.676$; right limb: $F=2.984, P=0.072$ ). The absolute grip strengths were 1206,1329 , and $1297 \mathrm{~g}$ for the Old, Trained, and Resveratrol rats, respectively, and were not significantly different among the three groups $(F=1.203, P=0.320)$ (Fig. 2A). However, the analysis of covariates showed significant differences among the three groups, with final body mass as a covariate $(F=6.488, P$ $=0.008)$. Similarly, the relative grip strengths were significantly higher in both the Trained group and Resveratrol group than in the Old group $(F=5.019, P=0.017)$ and were 2.04, 2.62, and $2.33 \mathrm{~g} \mathrm{~g}^{-1}$ for the Old, Trained, and Resveratrol rats, respectively (Fig. 2B).

Transcriptome sequencing and assembly

Three cDNA libraries were built from the skeletal muscle of each rat treatment. The parameters of the reads are shown in Table S1, and the reads have been uploaded to the NCBI Sequence Read Archive (accession number: SRA 605175). The expression level correlated strongly among treatments (Fig. 3).

Trained vs. Old: Only 21 differentially expressed genes were identified between the Trained group and the Old group (Table 3, Fig. S1), within which 14 genes (e.g., ribosomal RNA processing 7 homolog A (Rrp7a), RT1 class I, locus CE9, pseudogene 1 (RT1-CE9-ps1), the rRNA promoter binding protein (LOC257642), protein YIF1B (LOC103689986), and potassium sodium-activated channel subfamily $\mathrm{T}$ member 1 (Kcnt1)) were downregulated, while 7 genes 
194 (e.g., slingshot protein phosphatase 2 (SSH2), polypeptide N-acetylgalactosaminyltransferase 5 195 (GALNT5), RGD1562339 (RGD1562339), EFR3 homolog B (EFR3b), zinc finger protein 474 196 (ZFP474), mitochondrial carrier triple repeat 1 (MCART1), and cytidine deaminase-like 197 (LOC100909857)) were upregulated in the Trained group compared to those in the Old group. 198 Seventy GO enrichment terms were enriched in the Trained group vs. the Old group (Table S2).

The twenty most enriched GO terms were primarily associated with RNA metabolic processes and transmembrane transporters (Table 4). KEGG analysis identified several significantly upregulated pathways in the Trained group vs. Old group, including mucin-type O-Glycan biosynthesis, drug metabolism, and pyrimidine metabolism, but with only one differentially expressed gene (Table S4). The eight selected genes (Prkaa-2, FOXO3, IGF-1, TRIM67, IL-6, MGMT, and SCN5a) were verified by quantitative real-time PCR, within which only two genes (CASK and TRIM67) showed significant differences in the Trained group vs. the Old group (Fig. 4).

Resveratrol feeding vs. Old: Only 12 differentially expressed genes were identified between the Resveratrol group and the Old group (Table 3, Fig. S2), within which 2 genes were downregulated and 10 genes (e.g., aldehyde dehydrogenase 1 family, member A1 (ALDH1a1), tripartite motif-containing 67 (TRIM67), synaptotagmin 1 (SYT1), syntaxin-7-like (LOC100910446), synaptosomal-associated protein 25 (SNAP25), transthyretin (TTR), solute carrier family 17 member 7 (SLC17a7), neural EGFL-like 2 (NELL2), apolipoprotein C-I-like (LOC100910181) were upregulated in the Resveratrol group compared to those in the Old group. We found 219 GO terms that were enriched in the Resveratrol group vs. the Old group (Table S3). The most enriched GO terms were mainly associated with neurotransmitter transport and synaptic vesicle (Table 4). KEGG analysis identified several significantly upregulated pathways in the Resveratrol group vs. the Old group, including synaptic vesicle cycle, nicotine addiction, retinol metabolism, insulin secretion, retrograde endocannabinoid signaling, and glutamatergic synapse, but with only one differentially expressed gene (Table S4). Only the synaptic vesicle cycle pathway was significantly enriched with more than one differentially expressed gene (Table 5). Only CASK and TRIM67 showed significant differences between the Resveratrol group and the Old group by quantitative real-time PCR verification (Fig. 4).

\section{Discussion}




\section{Effects of exercise}

Even though exercise was suggested as a potential strategy in addressing age-related sarcopenia, previous studies obtained conflicting results on the effect of exercise on muscle mass and function. Many studies have found that exercise can ameliorate the aging-related loss of muscle mass and function and change muscle function-related gene expression (Sakamoto et al. 2005; Thomson and Gordon 2005; Rolland et al. 2008; Shefer et al. 2010; Cisterna et al. 2016). However, the present study showed no positive effects of exercise on muscle mass and absolute strength (Fig. 1, 2), which may result from heterogeneity in subject characteristics and the exercise type and intensity (Pattanakuhar et al. 2017). A moderate intensity and duration was used in the exercise training program in our study. More studies are needed to test whether exercise works better with stronger intensity or longer duration. In addition, our results showed that the relative grip strength of the Trained group increased compared to the Old group, which could be primarily due to the remarkable effect of exercise training on weight loss.

One explanation for the loss of muscle mass and function in aging rats is the reduced activities of AMPK, a fundamental regulator of energy metabolism (Salminen et al., 2011; Hardie et al., 2014; Hardman et al., 2014). It has been reported that exercise may lead to AMPK activation (Sakamoto et al. 2005). However, the exercise-induced phosphorylation status of AMPK was observed only in the soleus muscle but not in the plantaris muscle of aged rats (Thomson and Gordon 2005). Some studies found no significant exercise-induced upregulation of AMPK expression in the muscle of aged rats (Reznick et al. 2007). The present study showed no positive effects of exercise on the expression of muscle function-related genes, e.g., AMPK, FOX3, and IGF-1 (Fig. 1, 2, 4; Table 3). In addition, the expression levels of CASK, sodium and calcium channel regulation-related genes (Eichel et al., 2016; Nafzger and Rougier, 2017) were even reduced in the aged rats after exercise training.

Recently, sixteen genes have been identified as grip strength determining genes in humans (Willems et al. 2017); however, none of these genes was regulated in aged rats by exercise training in the present study. In fact, only a limited number of genes were upregulated in the Trained group compared to the Old group, and several transport- or transcript-related genes were even downregulated in the Trained group, e.g., RRP7a, RT1CE9ps1, and KCNT1. Our results suggest that exercise training has limited effects on the expression of genes in the skeletal muscle of aged rats. However, our results presented only data on the transcriptomic response. More 
256 studies on other levels, such as protein expression, metabolites and hormone dynamics, are

257 necessary to test the effects of exercise on aging-related sarcopenia.

258 Effect of resveratrol

259 Similar to the effects of exercise training, dietary resveratrol has remarkable effects on weight 260 loss in aged rats. Consistently, it has been reported that resveratrol (300 and $750 \mathrm{mg} / \mathrm{kg} / \mathrm{d}$ ) 261 reduced the body weight of rats aged 6 weeks during the 13-week feeding period (Williams et al. 262 2009). In addition, resveratrol $(400 \mathrm{mg} / \mathrm{kg} / \mathrm{d})$ reduced the body weight of mice aged 8 weeks 263 during the 9-week feeding period, which was attributed to the increase in energy expenditure 264 (Lagouge et al. 2006). However, resveratrol at a dose of $400 \mathrm{mg} / \mathrm{kg} / \mathrm{d}$ did not significantly affect 265 the body weight of rats during the 2-week feeding period (Momken et al. 2011), and resveratrol 266 at a dose of $60 \mathrm{mg} / \mathrm{kg} / \mathrm{d}$ did not affect body weight in mice aged 28 months during the 10-month 267 feeding period (Jackson et al. 2011). That the body weight was reduced by a relatively higher dose and longer period (Lagouge et al. 2006 and the present results) but not by a lower dose or shorter period (Jackson et al. 2011; Momken et al. 2011) suggests that the effects of dietary resveratrol on body mass depend on both its dose and duration of administration. Consistently, it has been suggested that the effects of resveratrol on in vitro muscle cell plasticity are dosedependent: low resveratrol doses promoted in vitro muscle regeneration and attenuated the impact of ROS, while high doses blocked the regenerative process (Bosutti and Degans 2015). degradation, and protect against cellular oxidative stress (Naylor 2009; Kaminski et al. 2012; Gordon et al. 2013). In the present study, dietary resveratrol did not change the muscle mass or absolute strength of the aged rats, and the apparent increase in the relative strength should be attributed to the weight loss effects of resveratrol in the aged rats. The effects of dietary resveratrol on skeletal muscle mass and function remain diverse in previous studies. Resveratrol reduced the aging-related declines in muscle mass and function of mice at 18 weeks of age (Murase et al. 2009; Dolinsky et al. 2012) but not of mice at 28 months of age (Jackson et al. 2011). Additionally, dietary resveratrol attenuated the decrease in muscle mass and strength caused by mechanical unloading in young rats ( 250 g) (Momken et al. 2011) but was unable to attenuate the reductions in muscle mass in aged rats (32 months) (Bennett et al. 2013). This finding implies that resveratrol may affect the skeletal muscle differently in the old individuals compared to the young ones and may have limited effects in attenuating sarcopenia. 
287

Resveratrol activates a number of signaling pathways, including the expression of IGF-1, AKT, AMPK, and SIRT1, in myoblasts (Barger et al., 2008; Murase et al. 2009; Naylor 2009; Park et al. 2012; Montesano et al. 2013; Ligt et al. 2015). However, our study showed no effects of dietary resveratrol on the expression of muscular function-related genes. Similarly, no effects of resveratrol on gene expression profiles were observed in normal mice, and it was explained that collecting tissue samples from the objectives fasted overnight may mask the effects of resveratrol on its target gene expression (Yoshino et al. 2012), which could be a reason for the unchanged expression of those genes in rats by resveratrol in our study, since the rats were fasted overnight before tissue sampling.

Interestingly, however, resveratrol induced the expression of several genes, including ALDH1a1, TRIM67, SYT1, SNAP25, TTR, SLC17a7, NELL2, LOC100910446, and LOC100910181 (Table 3). Among these genes, most of them (e.g., SYT1, LOC100910446, SNAP25, SLC17a7) are related to neuron synaptosome function (Tucker and Chapman 2002; Yoshihara and Montana 2004; Santos and Voglmaier 2009; Antonucci et al. 2016). NELL2, a gene involved in neural cell growth and differentiation, was also upregulated in aged mice fed with resveratrol. The enhanced expression of these genes suggests an upregulation of synaptic vesicle cycle signaling in aged mice fed with resveratrol. SLC17a7 encodes vesicular glutamate transporter 1 (VGLUT1), which is associated with the functions in transporting the excitatory neurotransmitter glutamate into synaptic vesicles and has important roles in the neuromuscular synapse in nervous systems and neuromuscular junctions of muscles (Varoqui et al. 2002; Kraus et al. 2004). Even though the functional significance of VGLUT1 has not been determined, VGLUT1 expression has been found to be altered by the aging process and several diseases (Jung et al. 2018). A recent study found that retinal VGLUT1 protein and gene expression were decreased in diabetic mice, indicating changes in the signal transmission from photoreceptors to bipolar cells and among postreceptoral neurons (Ly et al. 2014). This study also found that the decreased expression of VGLUT1 could not be normalized by metformin treatment (Ly et al. 2014). The positive effects of resveratrol on SLC17a7 expression imply a potential application of diabetic retinopathy intervention.

Another finding of the present study was that a tripartite motif family gene (TRIM67) was upregulated in aged mice fed resveratrol. The TRIM protein family has been implicated in many biological processes, including cell differentiation, apoptosis, transcriptional regulation and 
318 signaling pathways, and plays an important role in the broader immune response (McNab et al. 319 2011), especially in the restriction of infection by lentiviruses (Ozato et al. 2008). 320 Overexpression of TRIM proteins can increase the membrane repair capacity of muscular 321 dystrophy and restore muscle function and morphology (Alloush 2013; Dahl-Halvarsson et al. 322 2018). TRIM67 is highly expressed in the brain and regulates neuritogenesis, brain development, and behavior (Yaguchi et al. 2012; Boyer et al. 2018). Our results suggest that resveratrol has positive effects on the expression of TRIM67 in the muscle of aged rats, implying that the underlying mechanisms warrant further study.

\section{Conclusions}

In conclusion, the results present the global transcriptomic information involved in exercise and anti-oxidation interventions of skeletal muscle function in aged rats. Neither exercise training nor resveratrol feeding has a remarkable effect on skeletal muscle function and related gene expression in aged rats. Interventions with other exercise programs and resveratrol doses are needed, and studies at the protein level are also necessary to test whether exercise and resveratrol interventions are effective in addressing aging-related sarcopenia. However, both exercise training and resveratrol feeding have remarkable effects on weight loss, which is beneficial for suppressing exercise loads in the elderly. Our findings should be verified by future studies in human subjects.

We thank Ms. Yuxing Zhao and Die Pu for their help in animal care. We sincerely thank the anonymous reviewers for their helpful suggestions and comments.

\section{References}

Akune, T., Muraki, S., Oka, H., Yoshimura, N., Tanaka, S., Kawaguchi, H., and Nakamura, K. (2014). Association of physical activities of daily living with the incidence of certified need of care in the long-term care insurance system of Japan: the ROAD study. Journal of Orthopaedic Science, 19(3): 489-496. 
347

Alloush J, Weisleder N. TRIM proteins in therapeutic membrane repair of muscular dystrophy. JAMA Neurol, 2013, 70(7): 928-931.

Antonucci, F., Corradini, I., Fossati, G., Tomasoni, R., Menna, E. and Matteoli, M. SNAP-25, a known presynaptic protein with emerging postsynaptic functions. Frontiers in Synaptic Neuroscience, 2016, 8: 7-7.

Bano G, Trevisan C, Carraro S, Solmi M, Luchini C, Stubbs B, Manzato E, Sergi G, Veronese N. Inflammation and sarcopenia: A systematic review and meta-analysis. Maturitas, 2017, 96: $10-15$.

Barger J L, Kayo T, Vann J M, Arias E B, Wang J, Hacker T A, Wang Y, Raederstorff D, Morrow J D, Leeuwenburgh C, Allison D B, Saupe K W, Cartee G D, Weindruch R, Prolla T A. A low dose of dietary resveratrol partially mimics caloric restriction and retards aging parameters in mice. PLOS ONE, 2008, 3(6). e2264

Bennett B T, Mohamed J S, Alway S E. Effects of resveratrol on the recovery of muscle mass following disuse in the plantaris muscle of aged rats. PLOS ONE, 2013, 8(12): e83518.

Bosutti A, Degens H. The impact of resveratrol and hydrogen peroxide on muscle cell plasticity shows a dose-dependent interaction. Scientific Reports, 2015, 5(1): 8093-8093.

Boyer NP, Monkiewicz C, Menon S, Moy SS, Gupton SL. Mammalian TRIM67 functions in brain development and behavior. eNeuro, 2018, 5(3): ENEURO.0186-18.2018.

Cisterna B, Giagnacovo M, Costanzo M, Fattoretti P, Zancanaro C, Pellicciari C, Malatesta M. Adapted physical exercise enhances activation and differentiation potential of satellite cells in the skeletal muscle of old mice. Journal of Anatomy, 2016, 228: 771-781.

Dahl-Halvarsson M, Olive M, Pokrzywa M, Ejeskar K, Palmer R H, Uv A, Tajsharghi H. Drosophila model of myosin myopathy rescued by overexpression of a TRIM-protein family member. Proc Natl Acad Sci U S A. 2018, 115(28): E6566-E6575.

Deschenes, M. R. Effects of aging on muscle fibre type and size. Sports Medicine, 2004, 34(12): 809-824.

Dolinsky, V. W., and Dyck, J. R. Calorie restriction and resveratrol in cardiovascular health and disease. Biochimica et Biophysica Acta, 2011, 1812(11): 1477-1489.

Dolinsky, V. W., Jones, K. E., Sidhu, R. S., Haykowsky, M. J., Czubryt, M. P., Gordon, T., and Dyck, J. R. Improvements in skeletal muscle strength and cardiac function induced by resveratrol during exercise training contribute to enhanced exercise performance in rats. The 
Journal of Physiology, 2012, 590(11): 2783-2799.

Gordon, B. S., Diaz, D. C., and Kostek, M. C. Resveratrol decreases inflammation and increases utrophin gene expression in the $\mathrm{mdx}$ mouse model of Duchenne muscular dystrophy. Clinical Nutrition, 2013, 32(1), 104-111.

Hardie DG, Ashford ML. AMPK: regulating energy balance at the cellular and whole body levels. Physiology (Bethesda). 2014; 29:99-107.

Hardman SE, Hall DE, Cabrera AJ, Hancock CR, Thomson DM. The effects of age and muscle contraction on AMPK activity and heterotrimer composition. Exp. Gerontol. 2014; 55:120128.

Jackson J R, Ryan M, Alway S E. Long-term supplementation with resveratrol alleviates oxidative stress but does not attenuate sarcopenia in aged mice. Journals of Gerontology Series A, 2011, 66(7): 751-764.

Jung H Y, Yoo D Y, Park J H, Kim J W, Chung J Y, Kim D W, Won M H, Yoon Y S, Hwang I K. Age-dependent changes in vesicular glutamate transporter 1 and 2 expression in the gerbil hippocampus. Molecular Medicine Reports, 2018, 17(5): 6465-6471.

Kalinkovich, A., and Livshits, G. Sarcopenia-the search for emerging biomarkers. Ageing Research Reviews, 2015, 58-71.

Kaminski J, Lancon A, Tili E, Aires V, Demarquoy J, Lizard G, Michaille J I, Latruffe N. Dietary resveratrol modulates metabolic functions in skeletal muscle cells. Journal of Food and Drug Analysis, 2012, 20(suppl. 1): 398-401

Kraus T, Neuhuber W, Raab M. Vesicular glutamate transporter 1 immunoreactivity in motor endplates of striated esophageal but not skeletal muscles in the mouse. Neuroscience Letters, 2004, 360(1): 53-56.

Lagouge M, Argmann C A, Gerharthines Z, Meziane H, Lerin C, Daussin F, Messadeq N, Milne J, Lambert P, Elliott P, Geny B, Laakso M, Puigserver P,Auwerx J. Resveratrol improves mitochondrial function and protects against metabolic disease by activating SIRT1 and PGC-1alpha. Cell, 2006, 127(6): 1109-1122.

Liao, ZY, Chen JL, Xiao MH, Sun Y, Zhao YX, Pu D, Lv AK, Wang ML, Zhou J, Zhu SY, Zhao KX, and Xiao Q. The effect of exercise, resveratrol or their combination on Sarcopenia in aged rats via regulation of AMPK/Sirt1 pathway. Exp Gerontol. 2017; , 98:, 177-183. 
Ligt M De, Timmers S, Schrauwen P. Resveratrol and obesity: Can resveratrol relieve metabolic disturbances?. Biochimica et Biophysica Acta, 2015, 1852(6): 1137-1144.

Ly A, Scheerer M, Zukunft S, Muschet C, Merl J, Adamski J, de Angelis MH, Neschen S, Hauck SM, Ueffing M. Retinal proteome alterations in a mouse model of type 2 diabetes. Diabetologia, 2014, 57(1): 192-203.

McNab F W, Rajsbaum R, Stoye J P, O’Garra A. Tripartite-motif proteins and innate immune regulation. Current Opinion in Immunology, 2011, 23: 46-56.

Momken I, Stevens L, Bergouignan A, Desplanches D, Rudwill F, Chery I, Zahariev A, Zahn S, Stein TP, Sebedio JL, Pujos-Guillot E, Falempin $\quad M$, Simon $\quad C$, Coxam V, Andrianjafiniony T, Gauquelin-Koch G, Picquet F, Blanc S. Resveratrol prevents the wasting disorders of mechanical unloading by acting as a physical exercise mimetic in the rat. The FASEB Journal, 2011, 25(10): 3646-3660.

Montesano A, Luzi L, Senesi P, Mazzocchi N, Terruzzi I. Resveratrol promotes myogenesis and hypertrophy in murine myoblasts. Journal of Translational Medicine, 2013, 11(1): 310-310.

Murase T, Haramizu S, Ota N, Hase T. Suppression of the aging-associated decline in physical performance by a combination of resveratrol intake and habitual exercise in senescenceaccelerated mice. Biogerontology, 2009, 10(4): 423-434.

Naylor A J. Cellular effects of resveratrol in skeletal muscle. Life Sciences, 2009, 84(19): 637640.

Ozato K, Shin D M, Chang T H, Morse H C. TRIM family proteins and their emerging roles in innate immunity. Nat. Rev. Immunol., 2008, 8(11): 849-60.

Park S, Ahmad F, Philp A, Baar K, Williams T, Luo H, Ke H, Rehmann H, Taussig R, Brown A L, Kim M K, Beaven M A, Burgin A B, Manganiello V, Chung J H. Resveratrol ameliorates aging-related metabolic phenotypes by inhibiting cAMP phosphodiesterases. Cell, 2012, 148(3): 421-433.

Pattanakuhar S, Pongchaidecha A, Chattipakorn N, Leenders, M, Verdijk, L B, Van Loon L J, Parise G. The effect of exercise on skeletal muscle fibre type distribution in obesity: From cellular levels to clinical application. Obesity Research and Clinical Practice. 2017, 11(5): 112-132.

Reznick R M, Zong H, Li J, Morino K, Moore I K, Yu H J, Shulman G I. Aging-associated reductions in AMP-activated protein kinase activity and mitochondrial biogenesis. Cell 
440

Metabolism, 2007, 5(2): 151-156.

Rolland Y, Czerwinski S A, Van Kan G A, Morley J E, Cesari M, Onder G, Vellas B. Sarcopenia: Its assessment, etiology, pathogenesis, consequences and future perspectives. Journal of Nutrition Health and Aging, 2008, 12(7): 433-450.

Russ D W, Greggcornell K, Conaway M J, Clark B C. Evolving concepts on the age-related changes in "muscle quality". Journal of Cachexia, Sarcopenia and Muscle, 2012, 3(2): 95109.

Ryall J G, Schertzer J D, Lynch G S. Cellular and molecular mechanisms underlying age-related skeletal muscle wasting and weakness. Biogerontology, 2008, 9: 213-228.

Sakamoto K, McCarthy A, Smith D, Green K A, Hardie D G, Ashworth A, Alessi D R. Deficiency of LKB1 in skeletal muscle prevents AMPK activation and glucose uptake during contraction. EMBO J, 2005, pp. 1810-1820.

Salminen A, Hyttinen J M, Kaarniranta K. AMP-activated protein kinase inhibits NF-(B signaling and inflammation: impact on health span and lifespan. J Mol Med (Berl), 2011: 89: 667-676.

Santos M S, Li H, Voglmaier S M. Synaptic vesicle protein trafficking at the glutamate glutamate synapse. Neuroscience, 2009, 158(1): 189-203.

Shefer G, Rauner G, Yablonka-Reuveni Z, Benayahu D. Reduced satellite cell numbers and myogenic capacity in aging can be alleviated by endurance exercise. PLoS ONE, 2010, 5(10): e13307.

Sousa-Victor P, Muñoz-Cánoves P. Regenerative decline of stem cells in sarcopenia. Molecular Aspects of Medicine. 2016, 50:109-117.

Thomson D M, Gordon S E. Diminished overload-induced hypertrophy in aged fast-twitch skeletal muscle is associated with AMPK hyper phosphorylation. J. Appl. Physiol. 2005, 98: $557-564$.

Trapnell C, Williams B A, Pertea G, Mortazavi A, Kwan G, Van Baren M J, Pachter L. Transcript assembly and quantification by RNA-Seq reveals unannotated transcripts and isoform switching during cell differentiation. Nature Biotechnology, 2010, 28(5): 511-515.

Tucker W C, Chapman E R. Role of synaptotagmin in Ca2+-triggered exocytosis. Biochemical Journal, 2002, 366(1): 1-13.

Vang O, Ahmad N, Baile CA, Baur JA, Brown K, Csiszar A, Das DK, Delmas D, Gottfried 

C, Lin H Y, Ma Q Y, Mukhopadhyay P, Nalini N, Pezzuto J M, Richard T, Shukla Y, Surh Y J, Szekeres T, Szkudelski T, Walle T, Wu J M. (2011). What is new for an old molecule? Systematic review and recommendations on the use of resveratrol. PLoS ONE. 6 (6): e19881.

Varoqui H, Schäfer MK, Zhu H, Weihe E, Erickson JD. Identification of the differentiationassociated $\mathrm{Na}+/ \mathrm{PI}$ transporter as a novel vesicular glutamate transporter expressed in a distinct set of glutamatergic synapses. J Neurosci, 2002, 22: 142-155.

Willems S.M., Wright D.J., Day F.R. Scott R.A. Large-scale GWAS identifies multiple loci for hand grip strength providing biological insights into muscular fitness. Nature Communications 2017, 8: 16015.

Williams L D, Burdock G A, Edwards J, Beck M., Bausch J. Safety studies conducted on highpurity trans-resveratrol in experimental animals. Food and Chemical Toxicology, 2009, 47(9): 2170-2182.

Yaguchi H, Okumura F, Takahashi H, Kano T, Kameda H, Uchigashima M, Tanaka S, Watanabe M, Sasaki H, Hatakeyama S. TRIM67 protein negatively regulates Ras activity through degradation of 80K-H and induces neuritogenesis. J Biol Chem, 2012, 287: 1205012059.

Yoshihara M, Montana E S. The Synaptotagmins: Calcium Sensors for Vesicular Trafficking: The Neuroscientist, 2004, 10(6): 566-574.

Yoshino J, Conte C, Fontana L, Mittendorfer B, Imai S, Schechtman K B, Gu C, Kunz I, Rossi Fanelli F, Patterson B W, Klein S. Resveratrol supplementation does not improve metabolic function in nonobese women with normal glucose tolerance. Cell Metabolism, 2012, 16(5): 658-664.

Zhou J, Liao Z, Chen J, Zhao K, and Xiao Q. Integrated study on comparative transcriptome and skeletal muscle function in aged rats. Mechanisms of Ageing and Development, 2018, 169, 32-39. 


\section{Figure Captions}

499 Figure 1. Gastrocnemius muscle indexes of the hindlimbs of the control rats (Old) and the rats treated with exercise training (Trained) and resveratrol feeding (Resveratrol).

501 Data are presented as the mean \pm s.e.m.

502 Figure 2. Comparisons of the absolute grip strength (A) and relative grip strength (B) between 503 the control rats $(\mathrm{Old})$ and the rats treated with exercise training (Trained) and resveratrol 504 feeding (Resveratrol).

505 Data are presented as the mean \pm s.e.m. *: significantly different from the control rats $(\mathrm{P}<0.05)$.

506 Figure 3. Correlations among the expression level of the muscle tissue samples of the control rats (Old) and the rats treated with exercise training (Trained) and resveratrol feeding (Resveratrol).

a, b, c: The three replicate samples of each group.

Figure 4. Quantitative real-time PCR verifications for the selected genes of the control rats (Old) and the rats treated with exercise training (Trained) and resveratrol feeding (Resveratrol).

Data are presented as the mean \pm s.e.m. *: significantly different from the control rats $(\mathrm{P}<$ 513 $0.05)$. 
Table $\mathbf{1}$ (on next page)

Primers used in the qPCR. 
Table 1 Primers used in the qPCR.

\begin{tabular}{lll}
\hline Gene & Foward Primer (5'-3') & Reverse Premer (3'-5') \\
\hline FOXO3 & AACAGTACCGTGTTCGGACC & AGTGTCTGGTTGCCGTAGTG \\
CASK & CGAGCTTCCTGAGCACCGAG & CAGCGTGGAGGGTTCTGAAA \\
IGF-1 & CGTACCAAAATGAGCGCACC & CCTTGGTCCACACACGAACT \\
IL-6 & GCAAGAGACTTCCAGCCAGT & GTCTCCTCTCCGGACTTGTG \\
MGMT & AAAGCCGTCTGGCTGAAATTG & CCAGGACACATGCAGCTCTTTTAAT \\
PrKaa-2 & GATCGGACACTACGTGCTGG & ACTGCCACTTTATGGCCTGT \\
SCN5a & CTGACTATAGCCGCAGCGAA & GGCTCATCTGCAGAGCTAGG \\
TRIM67 & TCGTCCAGATGAAATTGCCAC & CCAGGGTGGCGCTGTTATTA \\
GAPDH & AACTCCCATTCCTCCACCTT & GAGGGCCTCTCTCTTGCTCT \\
\hline
\end{tabular}

2 


\section{Table 2 (on next page)}

Body mass parameters of the three groups of aged rats

Data were expressed as mean \pm s.e.m. ${ }^{\text {a,b,c }}$ : Different superscripts in each row indicate significant differences among groups $(P<0.05)$. Old: control aged rats; Trained: the aged rats treated with six weeks of exercise training; Resveratrol: the aged rats treated with six weeks of resveratrol feeding. 
1 Table 2 Body mass parameters of the three groups of aged rats.

\begin{tabular}{lccccc} 
Parameters & Old & Trained & Resveratrol & $F$ & $P$ \\
\hline $\begin{array}{l}\text { Initial body mass } \\
(\mathrm{g})\end{array}$ & $586.0 \pm 33.5$ & $591.1 \pm 30.3$ & $591.0 \pm 24.9$ & 0.010 & 0.990 \\
$\begin{array}{l}\text { Final Body mass } \\
(\mathrm{g})\end{array}$ & $596.6 \pm 34.7$ & $518.4 \pm 34.7$ & $559.5 \pm 26.9$ & 14.558 & $<0.0001$ \\
$\begin{array}{l}\text { Body mass gain } \\
(\mathrm{g})\end{array}$ & $10.6 \pm 11.5^{\mathrm{a}}$ & $-72.8 \pm 8.3^{\mathrm{c}}$ & $-31.5 \pm 12.4^{\mathrm{b}}$ & 14.663 & $<0.0001$ \\
$\begin{array}{l}\text { Specific growth } \\
\text { rate }\left(\% \text { day }^{-1}\right)\end{array}$ & $0.04 \pm 0.04^{\mathrm{a}}$ & $-0.31 \pm 0.03^{\mathrm{c}}$ & $-0.13 \pm 0.05^{\mathrm{b}}$ & 16.613 & $<0.0001$ \\
\hline
\end{tabular}

2 


\section{Table 3(on next page)}

Several differentially expressed genes in gastrocnemius muscle tissue of the three groups of aged rats.

Old: control aged rats; Trained: the aged rats treated with six weeks of exercise training;

Resveratrol: the aged rats treated with six weeks ofresveratrol feeding. 
1 Table 3 Several differentially expressed genes in gastrocnemius muscle tissue of the three groups 2 of aged rats.

\begin{tabular}{|c|c|c|c|}
\hline Gene symbol & Gene full name & $\begin{array}{l}\log _{2} \text { Fold } \\
\text { change }\end{array}$ & $\begin{array}{l}\text { Corrected- } \\
\text { P Value }\end{array}$ \\
\hline \multicolumn{4}{|l|}{ Trained vs Old } \\
\hline RRP7a & ribosomal RNA processing 7 homolog A & -2.623 & 0.000 \\
\hline RT1CE9ps1 & RT1 class I, locus CE9, pseudogene 1 & -2.453 & 0.050 \\
\hline LOC257642 & rRNA promoter binding protein & -2.005 & 0.050 \\
\hline LOC103689986 & protein YIF1B & -1.902 & 0.021 \\
\hline KCNT1 & $\begin{array}{l}\text { potassium sodium-activated channel } \\
\text { subfamily } T \text { member } 1\end{array}$ & -1.878 & 0.030 \\
\hline RRP8 & $\begin{array}{l}\text { ribosomal RNA processing } 8 \text {, } \\
\text { methyltransferase, homolog (yeast) }\end{array}$ & -1.563 & 0.005 \\
\hline DBP & D-box binding PAR bZIP transcription factor & -1.509 & 0.002 \\
\hline \multicolumn{4}{|l|}{ Up-regulated } \\
\hline $\mathrm{SSH} 2$ & slingshot protein phosphatase 2 & 1.330 & 0.021 \\
\hline GALNT5 & $\begin{array}{l}\text { polypeptide } \mathrm{N} \text { - } \\
\text { acetylgalactosaminyltransferase } 5\end{array}$ & 1.373 & 0.042 \\
\hline RGD1562339 & RGD1562339 & 1.647 & 0.050 \\
\hline EFR3b & EFR3 homolog B & 1.764 & 0.005 \\
\hline ZFPp474 & zinc finger protein 474 & 2.076 & 0.006 \\
\hline MCART1 & mitochondrial carrier triple repeat 1 & 2.549 & 0.019 \\
\hline LOC100909857 & cytidine deaminase-like & 3.260 & 0.005 \\
\hline $\begin{array}{l}\text { Resveratrol vs Old } \\
\text { Down-regulated }\end{array}$ & & & \\
\hline ZMYND19 & zinc finger, MYND-type containing 19 & -1.262 & 0.000 \\
\hline Up-regulated & & & \\
\hline ALDH1al & $\begin{array}{l}\text { aldehyde dehydrogenase } 1 \text { family, member } \\
\text { A1 }\end{array}$ & 1.213 & 0.000 \\
\hline TRIM67 & tripartite motif-containing 67 & 3.198 & 0.000 \\
\hline SYT1 & synaptotagmin 1 & 3.726 & 0.000 \\
\hline LOC100910446 & syntaxin-7-like & 4.571 & 0.000 \\
\hline SNAP25 & synaptosomal-associated protein 25 & 5.168 & 0.000 \\
\hline TTR & transthyretin & 5.377 & 0.000 \\
\hline SLC17a7 & solute carrier family 17 member 7 & 6.599 & 0.000 \\
\hline NELL12 & neural EGFL like 2 & Inf & 0.000 \\
\hline
\end{tabular}




\section{Table 4 (on next page)}

The most enriched Gene Ontology terms of the three groups of aged rats.

Old:control aged rats; Trained:the aged rats treated with six weeks of exercise training;Resveratrol:the aged rats treated with six weeks ofresveratrol feeding. 
1 Table 4 The most enriched Gene Ontology terms of the three groups of aged rats.

\begin{tabular}{|c|c|c|c|c|c|}
\hline GO accession & Description & Term type & P Value & Up & Down \\
\hline \multicolumn{6}{|c|}{ Trained vs. Old } \\
\hline GO:0034660 & ncRNA metabolic process & biological_process & 0.002697 & 0 & 3 \\
\hline GO:0006364 & rRNA processing & biological_process & 0.005946 & 0 & 2 \\
\hline GO:0016072 & rRNA metabolic process & biological_process & 0.00631 & 0 & 2 \\
\hline GO:0042254 & ribosome biogenesis & biological_process & 0.0165 & 0 & 2 \\
\hline GO:0034470 & ncRNA processing & biological_process & 0.017902 & 0 & 2 \\
\hline GO:0022613 & ribonucleoprotein complex biogenesis & biological_process & 0.035357 & 0 & 2 \\
\hline GO:0000139 & Golgi membrane & cellular_component & 0.026633 & 1 & 1 \\
\hline GO:0022857 & transmembrane transporter activity & molecular_function & 0.04874 & 0 & 3 \\
\hline \multicolumn{6}{|c|}{ Resveratrol vs. Old } \\
\hline GO:0006836 & neurotransmitter transport & biological_process & $7.01 \mathrm{E}-05$ & 3 & 0 \\
\hline GO:0001505 & regulation of neurotransmitter levels & biological_process & $7.68 \mathrm{E}-05$ & 3 & 0 \\
\hline GO:0042572 & retinol metabolic process & biological_process & $8.95 \mathrm{E}-05$ & 2 & 0 \\
\hline GO:0034308 & primary alcohol metabolic process & biological_process & 0.000159 & 2 & 0 \\
\hline GO:0048278 & vesicle docking & biological_process & 0.000258 & 2 & 0 \\
\hline GO:0016050 & vesicle organization & biological_process & 0.000291 & 3 & 0 \\
\hline GO:0001523 & retinoid metabolic process & biological_process & 0.000298 & 2 & 0 \\
\hline GO:0006066 & alcohol metabolic process & biological_process & 0.000356 & 3 & 0 \\
\hline GO:0016101 & diterpenoid metabolic process & biological_process & 0.000399 & 2 & 0 \\
\hline GO:0022406 & membrane docking & biological_process & 0.000415 & 2 & 0 \\
\hline GO:0006721 & terpenoid metabolic process & biological_process & 0.000528 & 2 & 0 \\
\hline GO:0031201 & SNARE complex & cellular_component & $2.35 \mathrm{E}-06$ & 3 & 0 \\
\hline GO:0008021 & synaptic vesicle & cellular_component & $3.31 \mathrm{E}-05$ & 3 & 0 \\
\hline GO:0060076 & excitatory synapse & cellular_component & $8.74 \mathrm{E}-05$ & 2 & 0 \\
\hline GO:0098793 & presynapse & cellular_component & 0.000105 & 3 & 0 \\
\hline GO:0030672 & synaptic vesicle membrane & cellular_component & 0.00026 & 2 & 0 \\
\hline GO:0042734 & presynaptic membrane & cellular_component & 0.000312 & 2 & 0 \\
\hline GO:0045202 & synapse & cellular_component & 0.000328 & 3 & 1 \\
\hline GO:0000149 & SNARE binding & molecular_function & $2.37 \mathrm{E}-05$ & 3 & 0 \\
\hline GO:0017075 & syntaxin-1 binding & molecular_function & $4.45 \mathrm{E}-05$ & 2 & 0 \\
\hline
\end{tabular}




\section{Table 5 (on next page)}

The significant KEGG enriched pathways the rats treated with six weeks of resveratrol feeding compared to the control rats.

Old: control aged rats; Resveratrol: the aged rats treated with six weeks ofresveratrol feeding. 
1 Table 5 The significant KEGG enriched pathways the rats treated with six weeks of resveratrol 2 feeding compared to the control rats.

\begin{tabular}{lllll}
\hline Pathway & KEGG ID & $\begin{array}{l}\text { Input } \\
\text { number }\end{array}$ & $\begin{array}{l}\text { Background } \\
\text { number }\end{array}$ & $\begin{array}{l}\text { Corrected } \\
\text { P-Value }\end{array}$ \\
\hline $\begin{array}{l}\text { Resveratrol vs Old up-regulated } \\
\text { Synaptic vesicle cycle }\end{array}$ & rno04721 & 3 & 63 & 0.000 \\
\hline
\end{tabular}

3 


\section{Figure 1}

Gastrocnemius muscle indexes of the hindlimbs of the rats.

Data are presented as the mean \pm s.e.m. Blue: left hindlimb; red: right limb.Old: control aged rats; Trained: the aged rats treated with six weeks of exercise training; Resveratrol: the aged rats treated with six weeks ofresveratrol feeding.

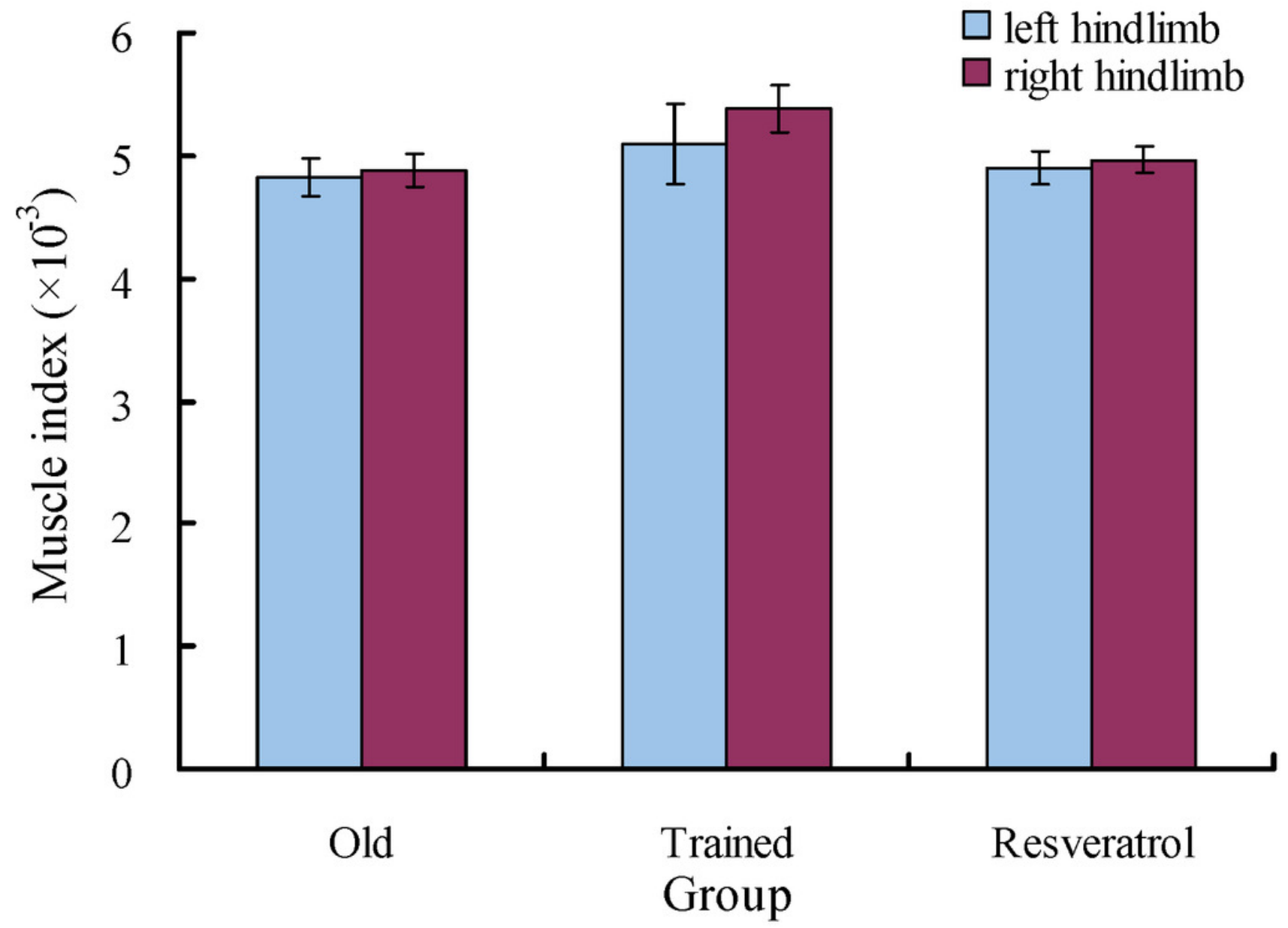




\section{Figure 2}

Comparisons of the absolute grip strength and relative grip strength between the control rats and the rats treated with exercise training and resveratrol feeding.

Data are presented as the mean \pm s.e.m. $*$ : significantly different from the control rats $(P<$ 0.05). A: the absolute grip strength; B: the relative grip strength. Old: control aged rats; Trained: the aged rats treated with six weeks of exercise training; Resveratrol: the aged rats treated with six weeks ofresveratrol feeding. 

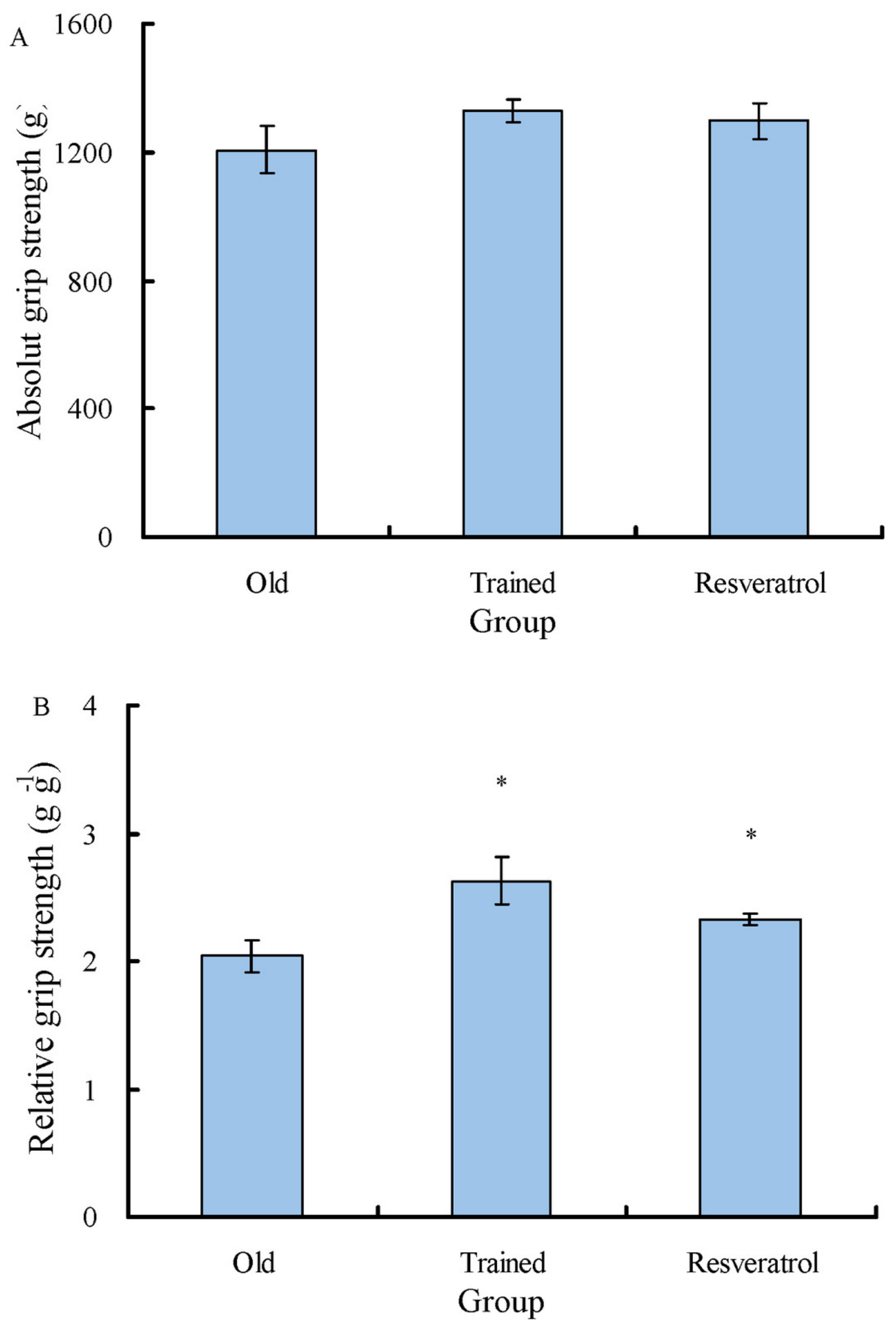


\section{Figure 3}

Correlations among the expression level of the muscle tissue samples of the control rats and the rats treated with exercise training and resveratrol feeding.

a, b, c: The three replicate samples of each group. Old: control aged rats; Trained: the aged rats treated with six weeks of exercise training; Resveratrol: the aged rats treated with six weeks ofresveratrol feeding.

\begin{tabular}{|c|c|c|c|c|c|c|c|c|c|c|}
\hline 1 & 0.98 & 0.99 & 0.99 & 0.99 & 0.98 & 0.99 & 0.98 & 0.99 & Old-a & \\
\hline 0.98 & 1 & 0.98 & 0.97 & 0.98 & 0.98 & 0.98 & 0.99 & 0.99 & Old-b & \\
\hline 0.99 & 0.98 & 1 & 0.98 & 0.98 & 0.98 & 0.99 & 0.99 & 0.99 & Old-c & \\
\hline 0.99 & 0.97 & 0.98 & 1 & 0.99 & 0.98 & 0.98 & 0.97 & 0.98 & Tra-a & \\
\hline 0.99 & 0.98 & 0.98 & 0.99 & 1 & 0.98 & 0.98 & 0.97 & 0.98 & Tra-b & -0.98 \\
\hline 0.98 & 0.98 & 0.98 & 0.98 & 0.98 & 1 & 0.98 & 0.98 & 0.98 & Tra-c & \\
\hline 0.99 & 0.98 & 0.99 & 0.98 & 0.98 & 0.98 & 1 & 0.98 & 0.99 & Res-a & \\
\hline 0.98 & 0.99 & 0.99 & 0.97 & 0.97 & 0.98 & 0.98 & 1 & 0.99 & Res-b & \\
\hline 0.99 & 0.99 & 0.99 & 0.98 & 0.98 & 0.98 & 0.99 & 0.99 & 1 & Res-c & \\
\hline $\begin{array}{l}\frac{\sigma}{\delta} \\
\frac{\dot{t}}{0}\end{array}$ & 竞 & 旁 & 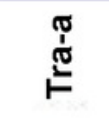 & 兽 & 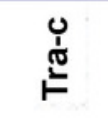 & 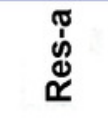 & 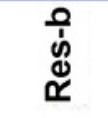 & 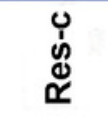 & & \\
\hline
\end{tabular}


Figure 4

Quantitative real-time PCR verifications for the selected genes of the control rats and the rats treated with exercise training and resveratrol feeding.

Data are presented as the mean \pm s.e.m. *: significantly different from the control rats $(\mathrm{P}<$ 0.05). Old: control aged rats; Trained: the aged rats treated with six weeks of exercise training; Resveratrol: the aged rats treated with six weeks ofresveratrol feeding.
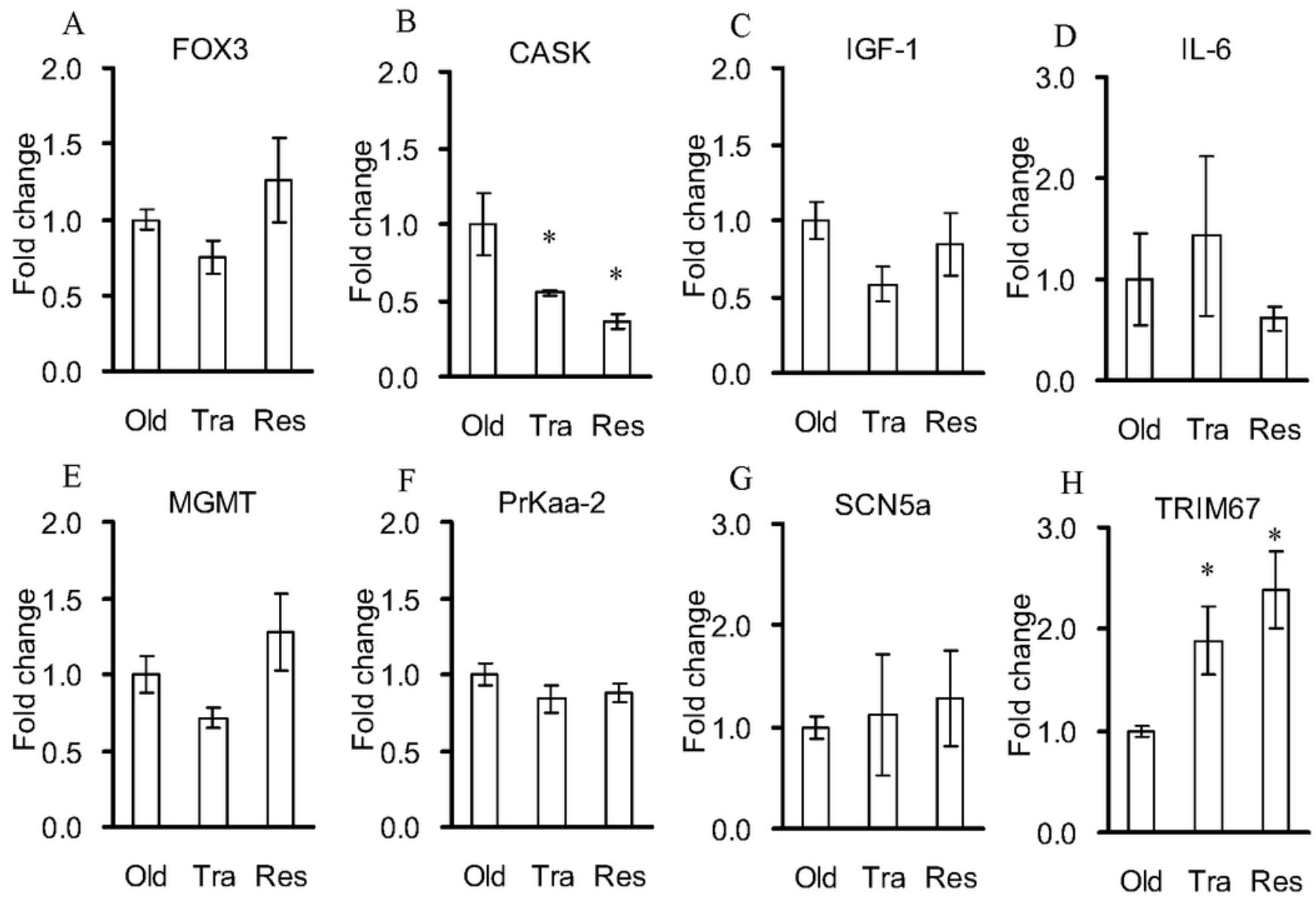